\title{
Factors Associated With Rapid Progression to Endstage Kidney Disease in Lupus Nephritis
}

\author{
Konstantinos Tselios ${ }^{1}$ (D), Dafna D. Gladman ${ }^{1}$ (D), Cameron Taheri ${ }^{1}$, Jiandong $\operatorname{Su}^{1}$, and Murray B. Urowitz ${ }^{1}$ (iD
}

\begin{abstract}
Objective. Lupus nephritis (LN) may lead to endstage kidney disease (ESKD) in 22\% of patients over a period of 15 years, with the risk being particularly higher in diffuse proliferative forms. The rate of kidney function decline varies. However, a catastrophic course leading to ESKD within a few years from onset is uncommon. The aim of the present study was to assess the factors associated with rapid progression to ESKD in patients with LN.

Methods. Patients from the Toronto Lupus Clinic with biopsy-proven LN at presentation and estimated glomerular filtration rate (eGFR) $60 \mathrm{~mL} / \mathrm{min} / 1.73 \mathrm{~m}^{2}$, who developed ESKD within 3 years were retrieved. Pathology reports were reviewed with particular emphasis on distinct histopathologic features. Demographic, clinical, laboratory, and therapeutic variables were also analyzed.

Results. Ten patients (1.8\% of the total LN population) developed ESKD within 3 years of diagnosis. Their mean age was $34.2 \pm 7.3$ years, mean time to ESKD $19.2 \pm 12.4$ months, initial eGFR $90.2 \pm 24.9 \mathrm{~mL} /$ $\mathrm{min} / 1.73 \mathrm{~m}^{2}$, proteinuria $2.7 \pm 1.04 \mathrm{~g} / 24 \mathrm{~h}$. The median rate of kidney function decline was $>43 \mathrm{~mL} /$ $\mathrm{min} / 1.73 \mathrm{~m}^{2} /$ year. One patient had LN class III, 5 had LN class IV, 2 had membranous LN (class V), and another 2 had mixed IV/V. Moreover, 2 patients had extensive thrombotic microangiopathy, 1 collapsing glomerulonephritis, and 1 concomitant antiglomerular basement membrane (anti-GBM) nephropathy. Four patients showed no unusual kidney pathology; all of them had severe noncompliance (discontinued all medications to follow alternative treatment).

Conclusion. Catastrophic progression to ESKD is uncommon in LN. The major associated factors are poor compliance and distinct histopathologic features such as thrombotic microangiopathy, collapsing glomerulopathy, and concomitant anti-GBM nephropathy.
\end{abstract}

Key Indexing Terms: endstage kidney disease, lupus nephritis, rapid progression

Lupus nephritis (LN) affects approximately $40 \%$ of patients with systemic lupus erythematosus (SLE) and may lead to endstage kidney disease (ESKD) in $22 \%$ over a period of 15 years ${ }^{1}$. The risk was particularly high (up to 44\%) in those with diffuse proliferative forms (class IV). Kidney injury is also the most important predictor of mortality in this population. Compared to non-SLE patients with ESKD, patients with LN on dialysis have a greater than 4-fold increased risk of death (95\% CI 1.2-15.2) ${ }^{2}$.

Although the rate of kidney function decline varies among patients, a "catastrophic" course defined as $\geq 20 \mathrm{~mL} /$ $\min / 1.73 \mathrm{~m}^{2} /$ year of the estimated glomerular filtration rate

The University of Toronto Lupus Program is funded in part by the University Health Network, Toronto General, and Toronto Western Research Foundation.

${ }^{1}$ K. Tselios, MD, PhD, D.D. Gladman, MD, FRCPC, C. Taheri, BHSc, J. Su, MB, MSc, M.B. Urowitz, MD, FRCPC, University of Toronto Lupus Clinic, Centre of Prognosis Studies in the Rheumatic Diseases, University Health Network, Toronto, Ontario, Canada.

The authors declare no conflicts of interest.

Address correspondence to Dr. M.B. Urowitz, MD, FRCPC, University of Toronto Lupus Clinic, Centre for Prognosis Studies in the Rheumatic Diseases, Toronto Western Hospital, 399 Bathurst St. 1E-410B, Toronto, ON M5T 2S8,Canada.Email:m.urowitz@utoronto.ca.

Full Release Article. For details see Reprints and Permissions at jrheum.org. Accepted for publication August 14, 2020.
(eGFR) is rather uncommon. The aim of the present study was to assess the factors associated with rapid (within 3 years of diagnosis) progression to ESKD in patients with LN and with initially normal or mildly impaired kidney function.

\section{MATERIALS AND METHODS}

Setting. The University of Toronto Lupus Clinic (UTLC) has currently enrolled 2008 patients since its establishment in 1970. All patients fulfilled the revised American College of Rheumatology criteria for SLE classification, or had 3 criteria and a supportive kidney biopsy ${ }^{3}$. Patients are followed regularly at 2- to 6-month intervals according to a standardized research protocol, which is regularly updated. Regarding $\mathrm{LN}$, the protocol captures the histopathologic class according to the International Society of Nephrology/Renal Pathology Society classification ${ }^{4}$ along with all relevant laboratory (serum creatinine, 24-h proteinuria, urinary sediment including hematuria and casts, titers of anti-dsDNA antibodies, and levels of complements $\mathrm{C} 3$ and $\mathrm{C} 4$, etc.) and therapeutic data [dose and type of immunosuppressives, glucocorticoids (GC), angiotensin-converting enzyme inhibitors (ACEI), angiotensin receptor blockers, etc.]. Moreover, associated factors such as hypertension (including blood pressure level and relevant treatment) and diabetes are documented in each visit.

All individuals have provided written informed consent for studies being conducted at the UTLC. This study was approved by the University Health Network Research Ethics Board (UHN/REB 11-0397).

Patient selection. For the purpose of the present study, the data of the UTLC patients with new-onset LN and normal or mildly impaired kidney function (eGFR $60 \mathrm{~mL} / \mathrm{min} / 1.73 \mathrm{~m}^{2}$ ) who developed ESKD (defined as the initiation 
of dialysis or eGFR $<15 \mathrm{~mL} / \mathrm{min} / 1.73 \mathrm{~m}^{2}$ for 2 consecutive clinic visits) within 3 years since LN diagnosis were retrieved. Time to ESKD was defined as the period from the kidney biopsy (index date) to the incident ESKD date. Study design. The medical records of the eligible patients were reviewed with particular emphasis on the distinct histopathologic features, including moderate-to-severe glomerular sclerosis, interstitial fibrosis, tubuloreticular inclusions (TRI), thrombotic microangiopathy, collapsing glomerulonephritis, and podocyte effacement. The medical records were also reviewed for reports of poor compliance in the clinic visits preceding ESKD.

\section{RESULTS}

Five hundred and sixty patients with biopsy-proven LN were retrieved from the database, 43 of whom developed ESKD. Seventeen patients developed ESKD within 3 years; 7 of them were excluded because their baseline kidney function was severely impaired $\left(\right.$ eGFR $\left.9-31 \mathrm{~mL} / \mathrm{min} / 1.73 \mathrm{~m}^{2}\right)$. Ten patients with initially normal or mildly impaired kidney function (eGFR $60 \mathrm{~mL} / \mathrm{min} / 1.73 \mathrm{~m}^{2} ; 1.8 \%$ of the total $\mathrm{LN}$ population) developed ESKD within 3 years of LN diagnosis. The demographic, histopathologic, and kidney function characteristics are given in Table 1. Five patients had elevated blood pressure at the time of diagnosis (> 130/80 mmHg), whereas none had diabetes.

In addition, 2 patients had extensive thrombotic microangiopathy (TMA), 1 in the context of catastrophic antiphospholipid syndrome (Figures 1A,B), 1 collapsing glomerulopathy (Figures 1C,D), and 1 concomitant antiglomerular basement membrane (anti-GBM) antibodies. Severe

Table 1. Demographic, histopathologic, and initial renal function characteristics of the patients.

\begin{tabular}{|c|c|}
\hline & Values \\
\hline Sex, female:male & $8: 2$ \\
\hline Age at LN diagnosis, yrs & $34.2 \pm 7.3$ \\
\hline Disease duration, yrs & $2.2 \pm 2.5$ \\
\hline \multicolumn{2}{|l|}{ Race/ethnicity, n } \\
\hline Black & 5 \\
\hline Hispanic & 2 \\
\hline White & 2 \\
\hline Asian & 1 \\
\hline \multicolumn{2}{|l|}{ LN histopathologic class } \\
\hline III & 1 \\
\hline IV & 5 \\
\hline $\mathrm{IV} / \mathrm{V}$ & 2 \\
\hline $\mathrm{V}$ & 2 \\
\hline Activity index & $5.7 \pm 4.9$ \\
\hline Chronicity index & $3.3 \pm 3.0$ \\
\hline Serum creatinine, $\mu \mathrm{mol} / \mathrm{L}$ & $82.1 \pm 15.5$ \\
\hline $\mathrm{eGFR}, \mathrm{mL} / \mathrm{min} / 1.73 \mathrm{~m}^{2}$ & $90.2 \pm 24.9$ \\
\hline Anemia, $\mathrm{n}(\%)^{\mathrm{a}}$ & $3(30 \%)$ \\
\hline Proteinuria, g/24h & $2.7 \pm 1.04$ \\
\hline Nephrotic syndrome, n (\%) & $5(50 \%)$ \\
\hline Serum albumin, $\mathrm{g} / \mathrm{L}$ & $32.5 \pm 3.7$ \\
\hline Hypertension, $\mathrm{n}(\%)^{\mathrm{b}}$ & $5(50 \%)$ \\
\hline Time to ESKD, months & $19.2 \pm 12.4$ \\
\hline Rate of renal function decline, $\mathrm{mL} / \mathrm{min} / 1.73 \mathrm{~m}^{2} / \mathrm{yr}$, median & 43.3 \\
\hline
\end{tabular}

${ }^{\text {a }}$ Defined as hemoglobin $<120 \mathrm{~g} / \mathrm{L}$ for females and $<130 \mathrm{~g} / \mathrm{L}$ for males. ${ }^{\mathrm{b}}$ Defined as blood pressure $>130 / 80 \mathrm{mmHg}$. eGFR: estimated glomerular filtration rate; ESKD: endstage kidney disease; LN: lupus nephritis. interstitial inflammation was detected in 2 patients ( 1 class IV, 1 class IV/V). Moderate-to-severe interstitial fibrosis and tubular atrophy (IFTA) was reported in 4 patients, while severe podocyte effacement was reported in 3, and severe TRI in another 2. Four patients showed no unusual kidney pathology.

Remission induction therapy included GC (mean daily prednisone dose $53.3 \pm 10 \mathrm{mg} /$ day, 6 patients received intravenous pulses of methylprednisolone), immunosuppressives [cyclophosphamide (CYC) in 4, mycophenolate mofetil (MMF) in 7, azathioprine in 2], rituximab in 2 patients, and therapeutic plasma exchange in the patient with LN IV/anti-GBM nephritis. Of the 10 patients, 9 were concomitantly treated with hydroxychloroquine and 5 with ACEI (ramipril 5-10 mg/day). All 4 patients without unusual histopathologic features had severe noncompliance based on self-report (discontinued all medications against medical advice in order to follow alternative treatment). The distinct histopathologic characteristics and therapeutic approach for each patient are shown in Table 2.

\section{DISCUSSION}

The progression of LN to ESKD has been associated with several factors including ethnicity, younger age, male sex, diffuse proliferative LN, impaired kidney function at diagnosis, nephrotic range proteinuria, poor response to immunosuppressive therapy, hypertension, diabetes, and obesity ${ }^{5}$. A catastrophic course to ESKD, however, is rather uncommon. This study demonstrates that certain histopathological features and poor compliance are the main associated factors.

Collapsing glomerulopathy (CG) affects the podocytes with notable pathological features including tuft collapse and visceral epithelial hypertrophy. Its prognosis is typically poor, with $50-100 \%$ of patients progressing to ESKD despite immunosuppressive treatment ${ }^{6}$. Detwiler, et al reported that 8 of 14 patients with CG progressed to dialysis in 15 months after diagnosis, while 3 had died of dialysis complications before that timepoint ${ }^{7}$. Due to its rarity, there is currently no evidence-based treatment for CG, and fewer than $10 \%$ of the patients respond to immunosuppressives $^{8}$. MMF has been described to be efficacious in isolated cases 9 .

LN with concomitant anti-GBM antibodies has been reported infrequently. Li, et al detected anti-GBM antibodies in 14 of 157 (8.9\%) Chinese patients with SLE ${ }^{10}$. All of them developed LN, and over a third were diagnosed with Goodpasture syndrome. The prognosis is not known, although the presence of such antibodies may contribute to further glomerular injury. The therapeutic approach is empiric and consists of GC, CYC, and plasma exchange therapy ${ }^{11}$.

Histologic features of TMA may be detected in up to $20 \%$ of patients with LN and have been associated with poor prognosis ${ }^{12}$. Patients with concomitant LN and TMA have a 6-fold greater risk of progression to ESKD compared to patients with LN alone ( $30 \%$ vs $5 \%$ ) within 5 years ${ }^{12}$. The use of anticoagulation in addition to conventional immunosuppressives seems promising. In a multicenter study of 97 patients with concomitant LN and TMA, anticoagulation achieved higher rates of complete renal response, especially in those with antiphospholipid antibodies ${ }^{13}$. In selected cases, eculizumab may be of benefit. 

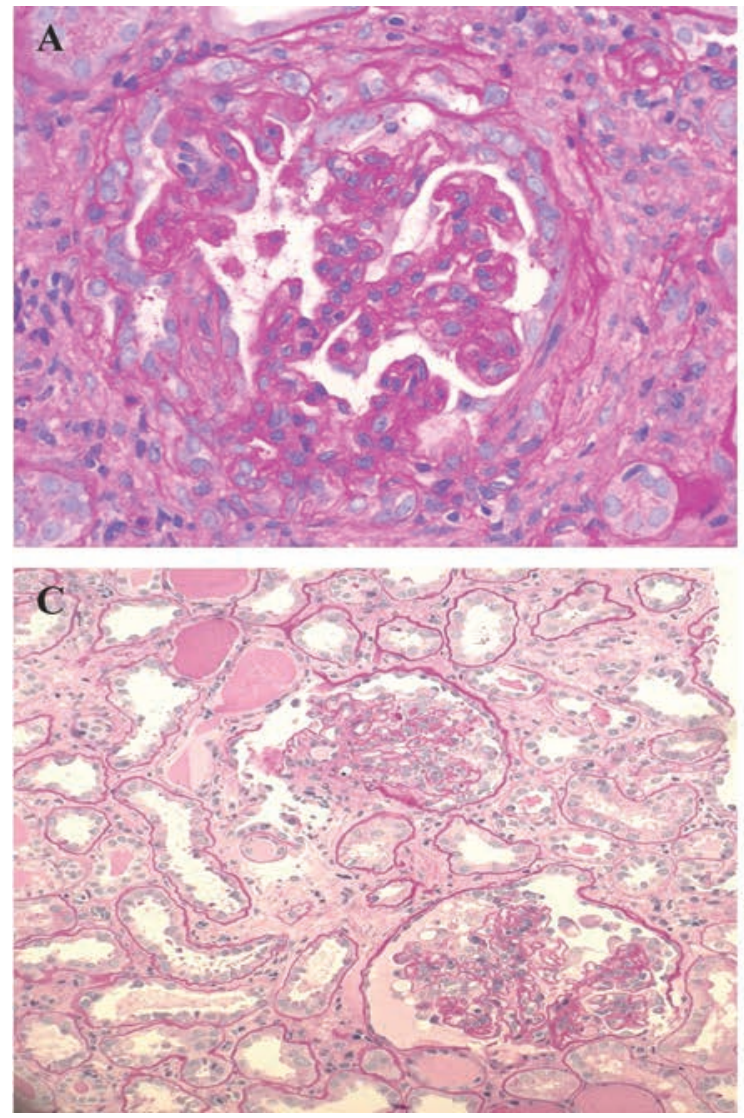
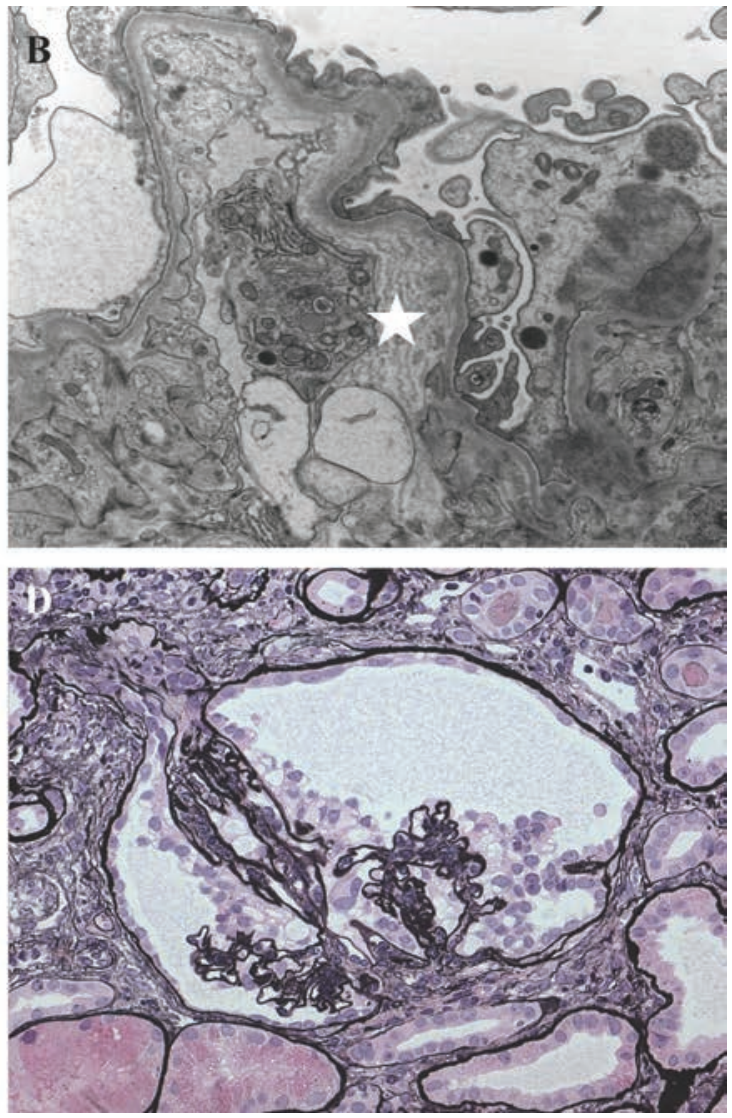

Figure 1. (A) Glomerulus displays endocapillary hypercellularity: Capillary loops are retracted; retraction and periglomerular fibrosis are seen; adhesions to the Bowman's capsule are identified; $40 \times$ PAS stain. (B) EM (same sample): Capillary loops show subendothelial widening (white star); there is endothelial swelling; findings are suggestive of thrombotic microangiopathy; EM direct magnification 12000×. (C) Two glomeruli displaying retraction of the glomerular tuft with hypertrophy and hyperplasia of the podocytes; PAS 20×. (D) EM (same sample): Marked retraction of the glomerular tuft with podocyte hypertrophy and hyperplasia; there is segmental scarring at the tip area (opposite to the vascular pole); findings are suggestive of collapsing glomerulopathy; EM direct magnification 12000×. EM: electron microscopy; PAS: Periodic acid-Schiff.

Park, et al reviewed 11 patients with concomitant LN and TMA (3 with antiphospholipid antibodies) who were refractory to GC, immunosuppressives, and plasma exchange ${ }^{14}$. Eculizumab was successful in 8 of 10 patients; of 7 patients who needed dialysis, 4 of them were off dialysis by the time of discharge.

Interstitial inflammation as well as IFTA are also associated with worse outcomes in LN. Severe interstitial inflammation (> 50\%) significantly increased the risk for ESKD as compared to mild disease $(<5 \%)$ in any $\mathrm{LN}$ class (HR 7.7, 95\% CI $3.8-15.7)^{15}$. That was particularly evident in LN IV (HR 14.1, $95 \%$ CI $4.5-44.1)$. Moreover, a combination of IFTA $>50 \%$ had a similar impact on the risk for ESKD compared to IFTA $<5 \%$ (HR 14, 95\% CI 4.9-39.8). Overall, there was a gradual decrease in kidney survival as interstitial inflammation or IFTA increased. The clinical significance of TRI is not known. In a recent report, $60 \%$ of the patients with TRI at kidney biopsy had LN, 20\% had chronic viral infections (hepatitis $B$, hepatitis $C$, and human immunodeficiency viruses), and another $20 \%$ had other diseases such as IgA nephropathy and Henoch-Schönlein purpura ${ }^{16}$. In a small series of $49 \mathrm{LN}$ patients, TRIs were detected in 12 and associated with class IV and increased activity index ${ }^{17}$.

Poor compliance was another factor to complicate patients with rapid progression to ESKD. In a previous systematic review, the percentage of nonadherent SLE patients ranged from $43 \%$ to $75 \%$, with most studies consistently reporting rates $>50 \%{ }^{18}$. The key determinants of nonadherence included depression, rural residence, lower education level, and polypharmacy. Bruce, et al also identified several patient-related factors contributing to the development of advanced chronic kidney disease (CKD) in SLE, including nonadherence due to potential adverse events, financial difficulties, or preference for alterative medications ${ }^{19}$.

Limitations of the present study are the small number of patients included and lack of a control group. As such, definitive conclusions regarding the effect of certain histologic features and nonadherence on the progression to ESKD cannot be drawn. However, some of these features (e.g., CG, TMA) are rare, and further study would require a multicenter collaboration. Moreover, the chronicity index was already elevated at diagnosis, implying the presence of kidney damage at the time of the biopsy. However, only 2 patients had a marginal eGFR $\left(61 \mathrm{~mL} / \mathrm{min} / 1.73 \mathrm{~m}^{2}\right)$ at the same time, indicating no significant CKD. The therapeutic approach was not standardized; as such, conclusions on the efficacy of any treatment should be cautiously interpreted. Moreover, genetic risk factors (such as the APOL1 allele $)^{20}$ were not assessed in our patients.

In conclusion, catastrophic progression to ESKD within 3 
Table 2. Main histologic and treatment characteristics in patients with catastrophic progression to ESKD.

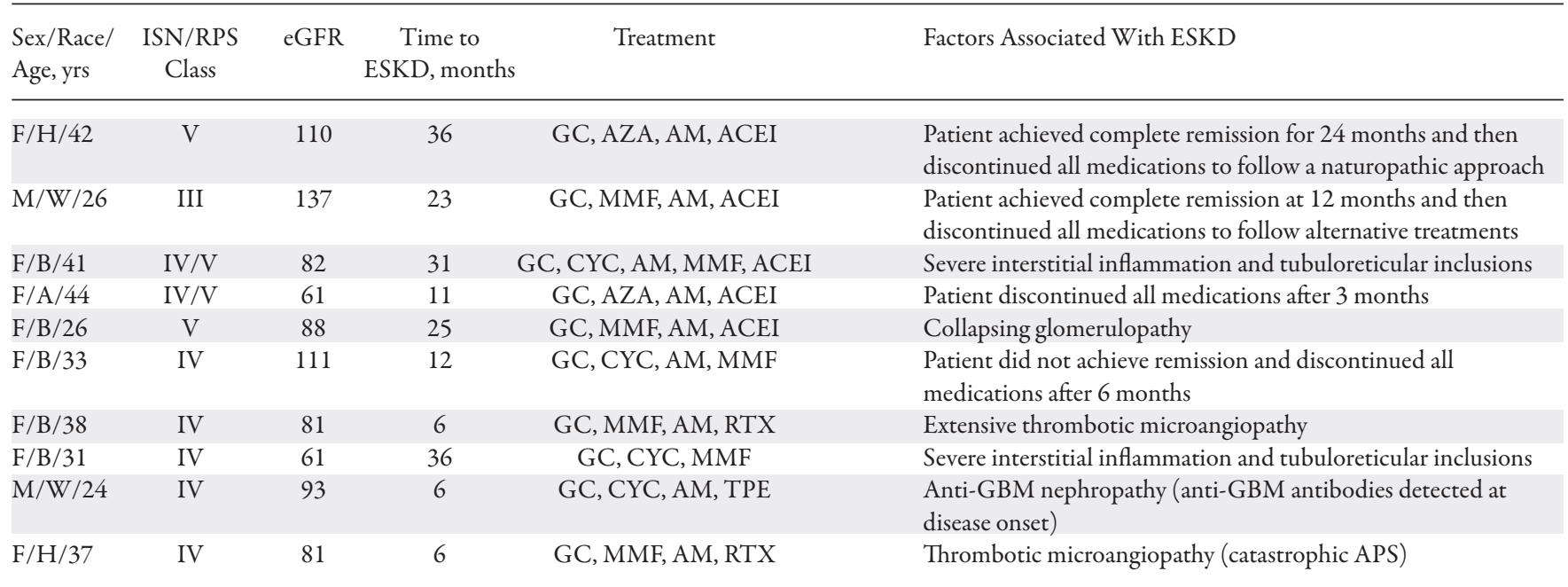

A: Asian patients; ACEI: angiotensin-converting enzyme inhibitor; AM: antimalarials; APS: antiphospholipid syndrome; AZA: azathioprine; B: Black patients; CYC: cyclophosphamide; eGFR: estimated glomerular filtration rate; ESKD: endstage kidney disease; F: female; GBM: glomerular basement membrane; GC: glucocorticoids; H: Hispanic patients; ISN/RPS: International Society of Nephrology/Renal Pathology Society; M: male; MMF, mycophenolate mofetil; RTX: rituximab; TPE: therapeutic plasma exchange; W: White patients.

years of diagnosis is uncommon in LN. The major potentially associated factors are distinct histopathologic features such as CG, anti-GBM antibodies, TMA, and severe interstitial inflammation. Poor compliance was also an aggravating factor in certain cases. Recognition of these features may stratify prognosis in the clinical setting and guide decisions for early intervention.

\section{ACKNOWLEDGMENT}

The authors would like to acknowledge Dr. Carmen Avila-Casado, Renal Pathologist and Director of the Electron Microscopy Laboratory of the University Health Network, University of Toronto, Toronto, Ontario, Canada, for her contribution and expertise regarding the renal pathology figures.

\section{REFERENCES}

1. Tektonidou MG, Dasgupta A, Ward MM. Risk of end-stage renal disease in patients with lupus nephritis, 1971-2015: a systematic review and Bayesian meta-analysis. Arthritis Rheumatol 2016;68:1432-41.

2. Lee PT, Fang HC, Chen CL, Chiou YH, Chou KJ, Chung HM. Poor prognosis of end-stage renal disease in systemic lupus erythematosus: a cohort of Chinese patients. Lupus 2003;12:827-32.

3. Hochberg MC. Updating the American College of Rheumatology revised criteria for the classification of systemic lupus erythematosus. Arthritis Rheum 1997;40:1725.

4. Weening JJ, D’Agati VD, Schwartz MM, Seshan SV, Alpers CE, Appel GB, et al. The classification of glomerulonephritis in systemic lupus erythematosus revisited. J Am Soc Nephrol 2004;15:241-50.

5. Maroz N, Segal MS. Lupus nephritis and end-stage kidney disease. Am J Med Sci 2013;346:319-23.

6. Schwimmer JA, Markowitz GS, Valeri A, Appel GB. Collapsing glomerulopathy. Semin Nephrol 2003;23:209-18.

7. Detwiler RK, Falk RJ, Hogan SL, Jennette JC. Collapsing glomerulopathy: a clinically and pathologically distinct variant of focal segmental glomerulosclerosis. Kidney Int 1994;45:1416-24.

8. Albaqumi M, Soos TJ, Barisoni L, Nelson PJ. Collapsing glomerulopathy. J Am Soc Nephrol 2006;17:2854-63.

9. Abadeer K, Alsaad AA, Geiger XJ, Porter IE. Collapsing glomerulopathy in systemic lupus erythematosus. BMJ Case Rep 2017;2017:bcr2016217840.
10. Li CH, Li YC, Xu PS, Hu X, Wang CY, Zou GL. Clinical significance of anti-glomerular basement membrane antibodies in a cohort of Chinese patients with lupus nephritis. Scand J Rheumatol 2006;35:201-8.

11. Yadla M, Krishnakishore C, Reddy S, Naveen PS, Sainaresh VV, Reddy MK, et al. An unusual association of anti-GBM diseases and lupus nephritis presenting as pulmonary renal syndrome. Saudi J Kidney Dis Transplant 2011;22:349-51.

12. Li C, Yap DYH, Chan G, Wen YB, Li H, Tang C, et al. Clinical outcomes and clinico-pathological correlations in lupus nephritis with kidney biopsy showing thrombotic microangiopathy. J Rheumatol 2019;46:1478-84

13. Sciascia S, Yazdany J, Dall'Era M, Fenoglio R, Radin M, Aggarwal I, et al. Anticoagulation in patients with concomitant lupus nephritis and thrombotic microangiopathy: a multicentre cohort study. Ann Rheum Dis 2019;78:1004-6.

14. Park MH, Caselman N, Ulmer S, Weitz IC. Complement-mediated thrombotic microangiopathy associated with lupus nephritis. Blood Adv 2018;2:2090-4.

15. Wilson PC, Kashgarian M, Moeckel G. Interstitial inflammation and interstitial fibrosis and tubular atrophy predict renal survival in lupus nephritis. Clin Kidney J 2018;11:207-18.

16. Lee CJ, Suh KS, Kim KH, Chang YK, Na KR, Lee KW. The clinicopathologic significance of endothelial tubuloreticular inclusions in glomerular diseases. Ultrastruct Pathol 2013;37:386-94.

17. Kfoury H. Tubulo-reticular inclusions in lupus nephritis: are they relevant? Saudi J Kidney Dis Transplant 2014;25:539-43.

18. Mehat P, Atiquzzaman M, Esdaile JM, Aviña-Zubieta A, De Vera MA. Medication non-adherence in systemic lupus erythematosus: a systematic review. Arthritis Care Res 2017;69:1706-13.

19. Bruce IN, Gladman DD, Urowitz MB. Factors associated with refractory renal disease in patients with systemic lupus erythematosus: the role of patient nonadherence. Arthritis Care Res 2000;13:406-8.

20. Freedman BI, Langefeld CD, Andringa KK, Croker JA, Williams AH, Garner NE, et al. End-stage renal disease in African Americans with lupus nephritis is associated with APOL1. Arthritis Rheumatol 2014;66:390-6. 